\title{
BENCH TEST RESULTS ON A NEW \\ TECHNIQUE FOR FAR-INFRARED POLARIMETRY
}

S. Barry, C. Nieswand

S.L. Prunty, H.M. Mansfield, P. O'Leary

submitted for publication to Review of Scientific Instruments 


\section{Bench Test Results on a New Technique for Far-Infrared Polarimetry}

S. Barry and C. Nieswand,

Centre de Recherches en Physique des Plasmas

Association EURATOM - Confédération Suisse

Ecole Polytechnique Fédérale de Lausanne,

Switzerland.

S. L. Prunty, H. M. Mansfield and P. O'Leary,

Department of Electrical Engineering and Microelectronics,

University College Cork,

IFA-EURATOM,

Ireland.

The results of bench tests performed on a new method of combined interferometry/polarimetry for the magnetic field reconstruction of tokamak plasmas is presented. In particular, the sensitivity obtained in the polarimetric measurement shows the feasibility of Faraday rotation determination approaching a precision of $\pm 0.2^{\circ}$. The method is based on an optically pumped far-infrared (FIR) laser with a rotating polarization where both the interferometric and polarimetric information is determined from phase measurements. Specific sources which introduce disturbances in the optical arrangement and which can limit the attainment of the polarimetric precision, mentioned above, are discussed. 
Interferometry is now a standard technique for the measurement of the line integrated electron density in magnetically confined plasmas. In order to maximise the information gathering capability many new and existing multi-channel interferometer systems are being modified to simultaneously measure the Faraday rotation angle of the probing beam thereby obtaining information regarding the current density profile. An important consideration of the upgrade is the minimisation of the investment in optical/electronic hardware.

Presently work is taking place to upgrade the FIR interferometer installed on TCV to a 14 channel interferometer/polarimeter system. It is planned to use the polarimeter data to improve the accuracy of the existing equilibrium reconstruction code $^{1}$. To prove a useful tool the TCV polarimeter was seen to require a precision of $\pm 0.2^{\circ}$ Faraday rotation.

Since the Faraday rotation method was first proposed by DeMarco and Segre ${ }^{2}$ for the measurement of magnetic field structure in tokamaks several techniques have been developed to measure the rotation of the plane of polarization of radiation traversing the plasma ${ }^{3}$.

Rice $^{4}$ proposed a scheme which permits existing interferometer devices to be readily converted to combined interferometer/polarimeter systems with a minimal investment in ancillary optical hardware. In fact, a single detector element per channel can perform the dual function of interferometric and polarimetric measurements. Rice's polarimeter scheme utilises a rotating elliptically polarized probing beam, produced by passing an elliptically polarized beam through a rapidly rotating half-wave plate. This is superimposed with a frequency shifted reference beam on the detectors. The resultant signals are modulated waveforms where the phase shift of the modulation 
envelope, with respect to that of a reference signal from a beam passing through free space, is proportional to the Faraday rotation angle. After demodulation and band-pass filtering, the resulting polarimeter signal is proportional to

$$
\cos \left(4 \omega_{\mathrm{r}} \mathrm{t}+2 \Psi\right)
$$

where $\omega_{\mathrm{r}}$ is the mechanical rotation frequency of the half-wave plate. The Faraday rotation angle, $\Psi$, can be extracted using a zero-crossing phase comparator. Similarly, the interferometer data is determined by measuring the phase of the carrier signal with respect to that of the reference signal. It has been shown, however, that the measured interferometer phase contains an additional time-varying phase term superimposed on the required interferometer data ${ }^{5}$. This term can be expressed as;

$$
\Delta \Phi=\arctan \left[\varepsilon \tan \left(2 \omega_{\mathrm{r}} \mathrm{t}+\Psi\right)\right]-\arctan \left[\varepsilon \tan \left(2 \omega_{\mathrm{r}} \mathrm{t}\right)\right]
$$

where, $\varepsilon$ is a measure of the ellipticity of the FIR beam. This additional term is not a plasma effect but the result of the polarization ellipse technique itself. This extra term can be effectively removed by low-pass filtering the interferometer data with the subsequent loss of time resolution. In a previous communication 6 we described a method based on the principle of a rotating polarization which incorporates the essential features of Rice's method but does not contain any additional time-dependent phase errors. Accordingly, we showed that the interferometer time resolution, which is set by the carrier frequency, can be preserved.

The method uses the combination of a rotating linearly polarised beam (known as the "A" beam) and a plain linearly polarised beam ("B" beam) as the probing beam and produces signals similar to those 
obtained using Rice's method. After demodulation and appropriate band-pass filtering, the polarimeter signal is proportional to ${ }^{6}$,

$$
\frac{\mathrm{A}}{\mathrm{B} \cos \Psi} \cos \left(2 \omega_{\mathrm{r}} \mathrm{t}+\Psi\right)
$$

where A and B are the beam amplitudes (Fig. 1), and $\Psi$ Faraday rotation. It has been assumed that the phase difference $\phi_{0}$ between the "A" and "B" beams has been set to 0 or a multiple of $\pi$ by fine adjustment of the "B" path length by means of a translator (Fig. 1). Note that the frequency of the modulation envelope is lower by a factor of 2 compared to Rice's method (see Eq. 1).

In order to test the sensitivity of the modified method the optical arrangement shown in Fig. 1 was employed. This set-up can also simulate Rice's method by simply blocking the path "B" and inserting a quarter-wave plate in front of the rotating half-wave plate. It should be noted that we employed a rotating grating and not a dual pumped laser system to generate the frequency shift corresponding to the carrier frequency. For plasma simulation we employed a half-wave plate mounted in a precision rotator which could be stepped through very small angular rotations. The laser beam was produced by a $\mathrm{CO}_{2}$ pumped FIR laser which operated at a wavelength of $214 \mu \mathrm{m}$. The carrier and modulation frequencies were set at $100 \mathrm{kHz}$ and $500 \mathrm{~Hz}$ respectively. The detectors used were liquid Helium cooled InSb hot electron bolometers.

The signals were electronically demodulated using a standard diode-resistor-capacitor arrangement and the resulting signals were band-pass filtered $(500 \mathrm{~Hz} \pm 100 \mathrm{~Hz}$ ). The Faraday rotation, $\Psi$ is extracted using a phase comparator circuit shown schematically in Fig. 2. The filtered demodulated signals are first passed though high-gain amplifiers followed by comparators producing a train of pulses whose 
width is proportional to $\Psi$. The pulse train is then low-pass filtered with a cut-off frequency of $100 \mathrm{~Hz}$. The final output of the polarimeter circuitry is a de voltage level proportional to $\Psi$.

Our initial efforts to achieve the required resolution were, however, frustrated by the presence of a dominant fluctuation on the polarimeter output which limited the resolution to approximately 1 degree. Investigations showed the rotating grating to be the source of this fluctuation. A separate experiment to assess the grating quality, which essentially measured the localized reflectivity, showed considerable variations around the perimeter of the grating. These variations were a consequence of the manufacturing process. The grating grooves were cut sequentially with the result that possible tooling wear caused localised non-uniformities causing poor reflection/diffraction efficiency at these positions. The problem was discussed with Dr. G. Dodel and his colleagues in the Institut für Plasmaforschung at the University of Stuttgart who supplied us with a new grating. The grooves on this new grating were machined randomly so as to distribute possible tooling errors in a uniform manner over the entire circumference. A similarly conducted test of grating quality showed a substantial improvement over the original.

With the new grating in place the optical arrangement (Fig. 1) was initially configured to simulate Rice's method. Fig. 3 shows a typical experimental output in which the precision half-wave plate was stepped though steps of 0.05 mechanical degrees which corresponds to a $0.1^{\circ}$ Faraday rotation step size. From this output we conclude that the Faraday angle can be measured to an accuracy of approximately $0.05^{\circ}$ or less. A power spectrum analysis showed that the residual noise on the polarimeter output was attributed largely to the rotating grating with a smaller contribution from the spindle. To achieve this noise 
level, good optical alignment and beam overlap were found to be essential. The modified method was then simulated and a similar polarimeter output acquired. The output is shown in Fig. 4 for Faraday rotation steps of $0.4^{\circ}$. Examination of this plot reveals the resolution in polarimeter measurement to be approximately $0.3^{\circ}$ which is quite close to the $0.2^{\circ}$ required.

The noise level on the polarimeter output is slightly larger than that in the case of Rice's method and is dominated by an additional noise component at low frequency in the region of 1 to $2 \mathrm{~Hz}$. It is suspected that this additional noise component has it's origin in the optical path where the "A" and "B" beam are superimposed, with the likely source being the turbine driven spindle which rotates the halfwave plate. Turbulence in the exhaust gas from both the bearing and the turbine of the spindle is a possible cause of phase shifts in the beam paths resulting in slowly varying time-dependent changes in $\phi_{0}$. Recall that $\phi_{0}$ is set to zero or a multiple of $\pi$ in advance of each measurement.

Accordingly, computer simulations were undertaken to determine the dependence of the polarimeter output on changes in $\phi_{0}$. These show that time variations in $\phi_{0}$ do contribute additional noise to the polarimeter output. It is difficult to put a precise figure on the maximum allowable variation in $\phi_{0}$ as the noise attributed to it is dependent on many different parameters including the Faraday angle, the frequency of the variations, the cut-off frequencies of the various electronic filters and the modulation depth of the signals. If, on the other hand, $\phi_{0}$ is time invariant, it can be accounted for in the calibration of the system. However, as previously mentioned ${ }^{6}, \phi_{0}$ introduces an extra phase term into the measured interferometer data. The error due to this additional term can be kept to within a few 
percent with a precision in the phase difference, $\phi_{0}$, of up to $20^{\circ}$. Experimentally, the noise on the polarimeter output increased significantly by intentionally introducing mechanical vibrations onto the optical components in the A-B beam paths. The aforementioned observations provide evidence to support the view that the air turbulence is the most likely factor preventing the attainment of the $\pm 0.2^{\circ}$ resolution for the modified method. Efforts are underway to redirect the air from the beam path, although this may prove to be a difficult task in the case of the bearing supply as the air emerges directly inside the shaft of the spindle which supports the rotating halfwave plate. The use of an electrically driven spindle capable of high speed operation is another possible solution. Nonetheless, the attainment of a polarimeter resolution for this modified technique, not significantly removed from the $\pm 0.2^{\circ}$ required, is sufficient encouragement to proceed with the implementation of the full 14 channel interferometer/polarimeter diagnostic on TCV.

In summary, the testing of a new polarimetric technique for magnetic field reconstruction in tokamak plasmas has been described. The method, based on a modification of Rice's method, is capable of achieving Faraday angle resolution approaching $\pm 0.2^{\circ}$. Our experimental findings with regard to the use of a rotating grating rather than a dual pumped laser for the frequency shift generation within the system should be of importance for other diagnosticians intending to install similar apparatus.

\section{Acknowledgements}

This work has been partially supported by the Swiss National Science Foundation and EURATOM. S. Barry would like to acknowledge EURATOM for financial support through a Research Training 
Fellowship. We would also like to thank Dr. G. Dodel for manufacturing the grating. We are very grateful for the technical assistance of M. O'Shea and M. O'Gorman.

${ }^{1}$ F. Hofmann and G. Tonetti, Nucl. Fusion 28, 1871 (1988).

2F. DeMarco and S.E. Segre, Plasma Phys. 14, 245 (1972).

3A.J.H. Donné, Rev. Sci. Instrum, 66, 3407 (1995).

${ }^{4}$ B.W. Rice, Rev. Sci. Instrum. 63, 5002 (1992).

5W.R. Geck, X. Qin, J. Liao, C.W. Domier and N.C. Luhmann, Jr., Rev. Sci. Instrum. 66, 860 (1995)

${ }^{6} \mathrm{~S}$.Barry, C. Nieswand, F. Bühlmann, S. Prunty and H. Mansfield, Rev. Sci. Instrum. 67, 1814(1996) 
FIG. 1. Illustration of the optical arrangement used during bench tests.

FIG. 2. Schematic diagram of the polarimeter demodulation and phase detection electronics.

FIG. 3. Polarimeter electronics output for Rice's method with steps of $0.1^{\circ}$ Faraday rotation.

FIG. 4. Polarimeter electronics output for the modified method with steps of $0.4^{\circ}$ Faraday rotation. 


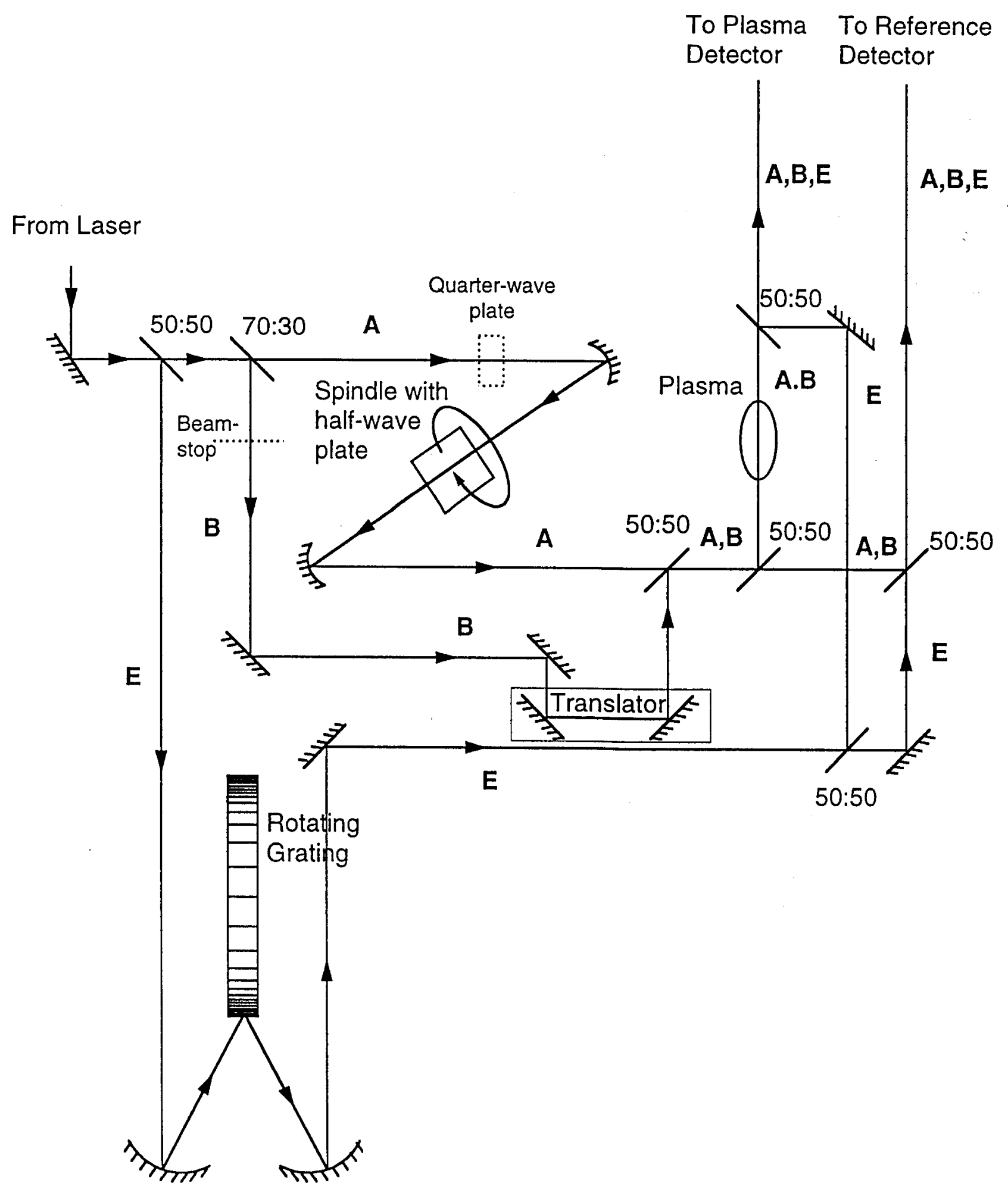

Fig. 1 


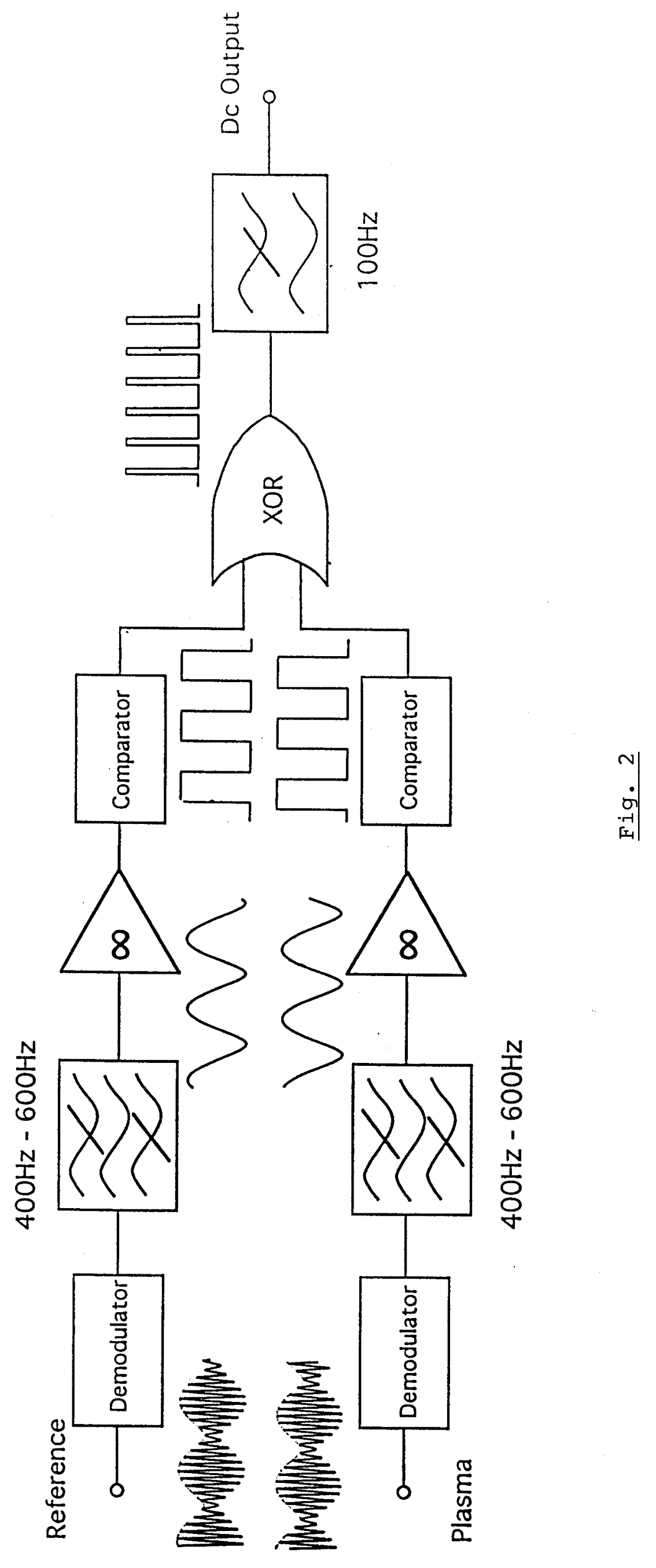




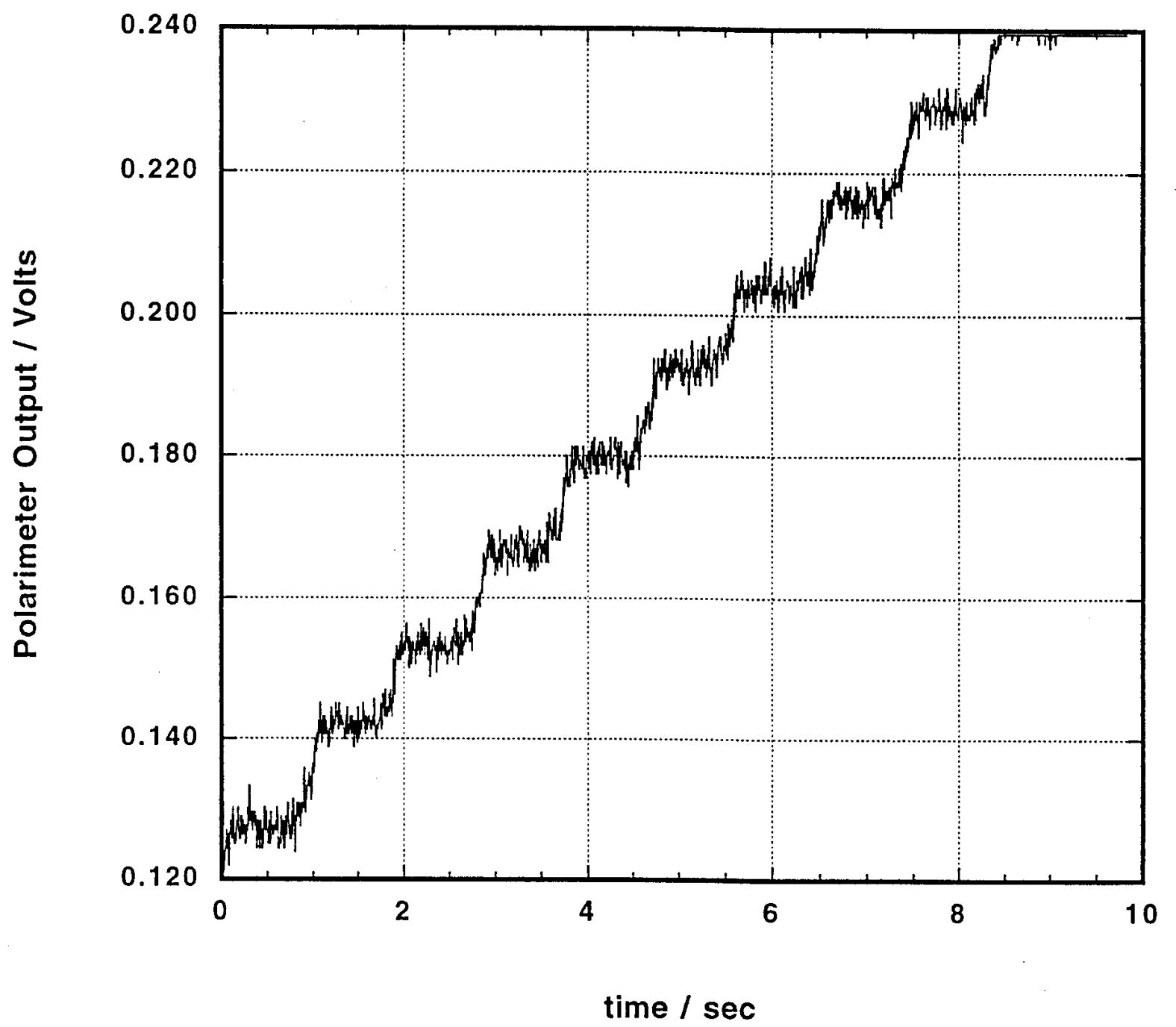

Fig. 3 


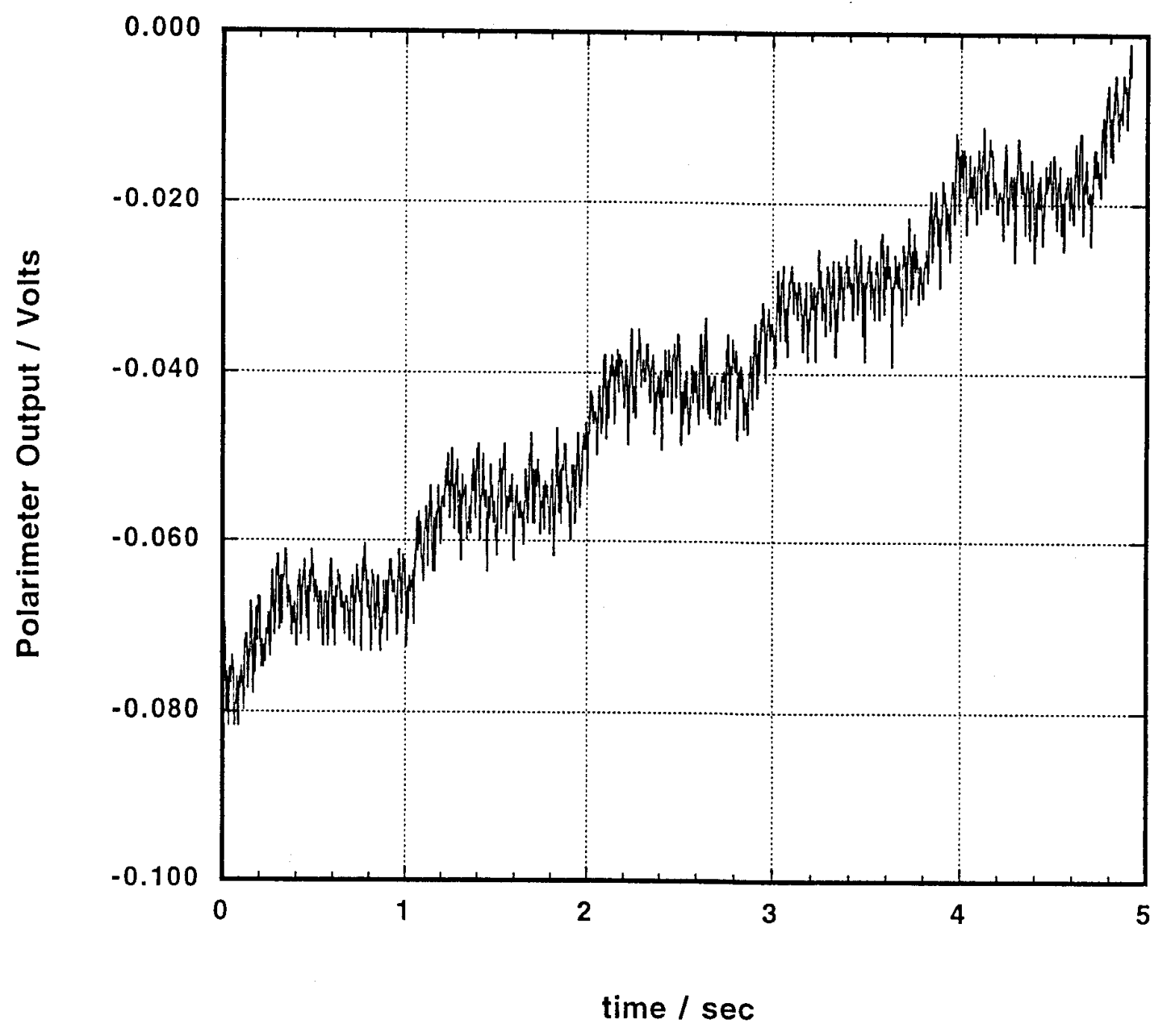

Fig. 4 